\title{
Data Pouring and Buffering on the Road: A New Data Dissemination Paradigm for Vehicular Ad Hoc Networks
}

\author{
Jing Zhao, Student Member, IEEE, Yang Zhang, Student Member, IEEE, and Guohong Cao, Senior Member, IEEE
}

\begin{abstract}
Vehicular ad hoc networks (VANETs) have recently received considerable attention. To support VANET-based applications, it is important to disseminate data from an information source (data center) to many vehicles on the road. Although disseminating data from a server to a large number of clients has been studied in the database community and the network community, many unique characteristics of the VANET bring out new research challenges. In this paper, we propose a data pouring (DP) and buffering paradigm to address the data dissemination problem in a VANET. In DP, data are periodically broadcast to vehicles on the road. In DP with intersection buffering (DP-IB), data poured from the source are buffered and rebroadcast at the intersections. We provide analytical models to explore the dissemination capacity (DC) of the proposed schemes. The analytical models also provide guidelines on choosing the system parameters to maximize the DC under different delivery ratio requirements. Simulation results show that the proposed DP-IB scheme can significantly improve the data delivery ratio and reduce network traffic.
\end{abstract}

Index Terms-Ad hoc networks, broadcasting, data dissemination, dissemination capacity, vehicular networks.

\section{INTRODUCTION}

$\mathbf{V}$ EHICULAR ad hoc networks (VANETs) have been envisioned to provide increased convenience and efficiency to drivers on the road [1]-[3]. For example, an alert message about a traffic accident or traffic jam can be propagated tens of miles along the road to help drivers select a better route. Department stores can disseminate sale advertisements to vehicles within the vicinity to attract consumers and to provide dining and parking information.

Through these applications, we can see that the VANET is very useful for disseminating data from an information source (data center) to many vehicles on the road. Although disseminating data from a server to a large number of clients has been studied in the database community and the network community [4], [5], many unique characteristics of the VANET bring out new research challenges. First, due to fast vehicle movement, the link topology changes rapidly [6]-[8]. As a result, many

Manuscript received February 15, 2007; revised May 30, 2007 and July 16, 2007. This work was supported in part by the National Science Foundation under Grant CNS-0092770, Grant CNS-0519460, and Grant CNS-0721479. The review of this paper was coordinated by Dr. L. Cai.

The authors are with the Department of Computer Science and Engineering, The Pennsylvania State University, State College, PA 16802 USA (e-mail: jizhao@cse.psu.edu; yangzhan@cse.psu.edu; gcao@cse.psu.edu).

Color versions of one or more of the figures in this paper are available online at http://ieeexplore.iee.org.

Digital Object Identifier 10.1109/TVT.2007.906412 well-studied structures for efficient data dissemination, such as trees, clustering, and grids, are extremely hard to set up and maintain. Second, the conventional broadcast mechanism for data dissemination may lead to broadcast storm [9] because the network node density is usually quite high in an urban area and extremely dense during rush hours or traffic jams. Third, the vehicle mobility is partially predictable since it is limited by the traffic pattern and the road layout [10]. Data dissemination techniques should address these unique characteristics of the VANET.

Recently, researchers have begun to address data dissemination issues in the VANET. $\mathrm{Xu}$ et al. propose an opportunistic dissemination (OD) scheme [11] that is similar to gossip [12]-[14]. In this approach, the data center periodically broadcasts some data, which will be received and stored by passing vehicles. Whenever two vehicles move into the transmission range of each other, they exchange data. This scheme does not rely on any infrastructure and, hence, is suitable for highly dynamic VANETs. However, after a data item has been propagated into the network, it is hard to timely remove the outdated information, particularly when it is frequently updated. In addition, the performance of the OD scheme is poor in areas with high vehicle density due to media access control (MAC)layer collisions [15]. This can easily lead to severe congestion and significantly reduce the data delivery ratio. To mitigate the excessive transmissions and congestion, Korkmaz et al. [16] propose a link-layer broadcast protocol to help disseminate the data. Theprotocol relies on link-layer acknowledge mechanisms to improve the reliability of the multihop broadcast. More specifically, only one vehicle is used to forward and acknowledge the broadcast packet to reduce the broadcast storm problem. However, in the case of network congestion, the link-layer solution is not enough. Furthermore, since many information sources may exist in a given urban area, the amount of broadcasted data from these sources can easily consume the limited bandwidth. Thus, it is important to study the maximum amount of data that can be disseminated in a given area [i.e., the dissemination capacity $(D C)]$.

In this paper, we propose a data pouring (DP) and buffering paradigm to address the data dissemination problem in a VANET. The proposed solution can reliably disseminate the data, efficiently utilize the limited bandwidth, and maximize the DC. In DP, data are periodically broadcasted to the vehicles on the road. As data are poured along the roads, they are delivered not only to the vehicles on these roads but also to the vehicles on 


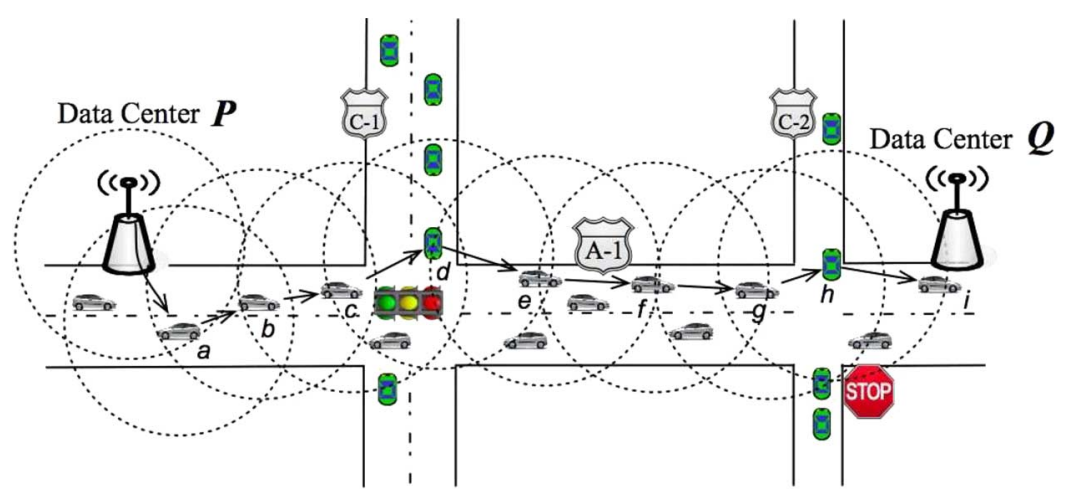

Fig. 1. Directional broadcast.

the intersecting roads when they move across the intersections. To further improve the performance, we propose an improved DP scheme, called DP with intersection buffering (DP-IB), which tries to reduce the amount of data poured from the source by buffering and rebroadcasting data at the intersection.

Periodically pouring data on the road is necessary since vehicles receiving the data may move away quickly, and vehicles coming later still need the data. With DP, the data are consistently available for vehicles crossing the dissemination area. In case there are a large amount of data from many information sources to disseminate, it is important to increase the amount of data that can be disseminated on a given road. Thus, we provide analytical models to explore the DC of the proposed schemes. The analytical models also provide guidelines on choosing the system parameters to maximize the DC under different delivery ratio requirements. Extensive simulations are provided and used to evaluate the proposed methodology. Simulation results show that the proposed DP-IB scheme can significantly improve the data delivery ratio and reduce network traffic.

The rest of this paper is organized as follows: Section II describes the DP and buffering schemes. Section III presents an analytical model to optimize the system parameters of our DP schemes. Performance evaluations are presented in Section IV. Section V concludes this paper.

\section{DP AND BUFFERING ON THE ROAD}

In this section, we first describe our system model and then present the DP and buffering schemes.

\section{A. System Model}

A VANET can be used to disseminate information about traffic accidents or traffic jams to help drivers select better routes. It can also be used to disseminate sale advertisements to attract customers. One way of achieving this goal is to have a data center disseminate the data. The data center can be a computer with a wireless interface, a wireless access point, or an infostation [17]. A data center may have a list of data items to disseminate, referred to as the dissemination data set (D-Set). The data center periodically broadcasts the D-Set so that each data item is broadcast once in each cycle. The disseminated data are relayed by moving vehicles and are poured to the desired area.
The data delivery information such as source id, source location, packet generation time, propagation direction, etc., is specified by the data center and placed in the packet header. The disseminated data are often spatial or/and temporal sensitive. For example, the traffic jam at downtown is not likely to be the interest of drivers 30 miles away and will also be less helpful $2 \mathrm{~h}$ later. Thus, a data item is attached with two more attributes: 1) the dissemination zone (D-Zone), which is defined as a rectangle area, and 2) the expiration time, which specifies the duration when the data item is valid. Vehicles outside of this D-Zone will discard the data to save bandwidth.

We assume that vehicles communicate with each other and with the data center through a short-range wireless channel (100-250 m). A vehicle knows its location by triangulation or through Global Positioning System device, which is already popular in new cars and will be common in the future. Vehicles use periodic beacon messages to report their moving velocity, direction, and location, so each vehicle (including the data center) can obtain the information about their one-hop neighbors and construct a neighbor list. To avoid overloading the channel with too many beacons, a vehicle can adjust its beacon interval based on its moving velocity, e.g., two beacons per second when moving over $40 \mathrm{mi} / \mathrm{h}$ and one beacon every $10 \mathrm{~s}$ when moving below $5 \mathrm{mi} / \mathrm{h}$. Techniques to achieve this can be found in [18] and [19].

\section{B. $D P$}

In this section, we first introduce the basic idea of the DP scheme and then propose solutions to make it more reliable.

1) Basic Idea: The DP scheme makes use of the partially predictable vehicle mobility limited by the road layout. Instead of spreading data throughout the network, it broadcasts the data to one or several roads, called axis roads (A-Roads). The A-Roads are selected from those main roads going through the data center, and they normally have a higher vehicle traffic density than other roads. The DP scheme also delivers data to vehicles moving on the roads that intersect with the A-Road, called crossing roads (C-Roads). However, it does not proactively push data to the C-Roads. Since vehicles on the C-Roads moving toward the A-Roads will eventually reach a point intersecting with A-Roads, they will obtain the data. Therefore, the D-Zone of a data item only includes the A-Road where this data item is propagated to. Fig. 1 shows the basic idea of the DP 
scheme. Both data centers $\mathrm{P}$ and Q select the horizontal road A-1 as the A-Road and the vertical roads C-1 and C-2 as the C-Roads. The data are broadcast along Road A-1. Fig. 1 only shows the data broadcasted from the data center P; the circles represent its broadcast coverage, which only covers Road A-1. Vehicles on Roads C-1 and C-2 receive the data when they go through the intersections.

In the DP scheme, the data center specifies the road to propagate data based on D-Zone and adds this information to the packet header. Then, it designates a passing-by vehicle to broadcast the data (for example, vehicle a in Fig. 1). To propagate the data to the desired road, the data item needs to be consecutively broadcast along the road by other vehicles such as $\mathrm{a} \rightarrow \mathrm{b} \rightarrow \mathrm{c} \rightarrow \mathrm{d} \rightarrow \mathrm{e} \rightarrow \mathrm{f} \rightarrow \mathrm{g} \rightarrow \mathrm{h}$ in Fig. 1. To deal with the broadcast storm problem [9], each designated broadcasting vehicle selects one vehicle that is farther away in the data propagation direction from its neighbor list and designates the selected neighbor as the next broadcast node by adding it to the packet header. After receiving the data, the designated vehicle (the forwarder) rebroadcasts the data. In this way, the data are poured to vehicles on the A-Roads. A forwarder delivers the data to all potential receivers within its one hop range and designates one vehicle as the next forwarder to broadcast the data father along the propagation direction.

Data invalidation [20] in the DP scheme is simple because vehicles do not cache the data. If the disseminated data are updated, the data center broadcasts the updated version, and vehicles will obtain the updated data.

2) Reliable $D P(R-D P)$ : The broadcasted data may be lost due to interference, packet collisions, and hidden-node problem. To ensure vehicles receiving the disseminated data, vehicles use request to send/clear to send (RTS/CTS) handshakes to reduce collisions and hidden-node problem. To make the broadcast more reliable, after the broadcasting, the sender holds the data packet for a short period of time in case retransmissions are needed. The rebroadcast from the next forwarder is used as an implicit acknowledge to the previous forwarder. If the sender does not hear the rebroadcast from the next forwarder within a prespecified time period, it selects another neighbor as the next forwarder and rebroadcasts the data.

When the sender is waiting for the rebroadcast by the next forwarder, it may receive some other broadcasted data toward the same direction. In this case, the sender has to buffer the data until the pending acknowledgement has been received from the next forwarder. There are two reasons for blocking the packet forwarding. First, if the selected next forwarder does not broadcast within a given period of time, it may indicate that there is an error on the selected next forwarder; e.g., the sender has selected an incorrect next forwarder or the selected forwarder is no longer within the transmission range. This may happen if the neighbor list is outdated or the sender misestimate the position of the selected forwarder. As a result, it should choose a different forwarder. Second, even if the next forwarder is correct, it may not be able to rebroadcast when the broadcasting load is too high. Adding more traffic may create congestion and result in more packet loss.

The R-DP scheme can improve the data delivery ratio even if data are disseminated many hops away from the data center.
However, it complicates the transmission with more control messages and backoff procedures, reducing the broadcast throughput. For example, when a large amount of data are independently poured from many data centers, the data collision probability is very high. The reliable transmission mechanisms will be frequently used, and a lot of bandwidth will be wasted by backoff timers, control messages, and RTS/CTS handshake. This will affect the DC.

3) $D C$ : One desired goal of data dissemination is to maximize the DC while ensuring a good data delivery ratio. Simply increasing the D-Set size is not an effective way to increase the DC since most data may not be successfully delivered to vehicles if the D-Set size is too large.

Data delivery is subject to two constraints. The first constraint is the data broadcast cycle, i.e., the time interval between broadcasting the same data item on an axial road. To deliver data to moving vehicles, the data are periodically broadcasted. If a vehicle is on the A-Road, it will receive the disseminated data sooner or later. However, vehicles on the C-road can only obtain the broadcasted data during a short time period, i.e., when they go cross the intersection. During this time period $t_{i}$, the vehicle should stay inside the wireless coverage centered at the intersection. Thus, $t_{i}$ is decided by the wireless coverage and the speed of the moving vehicle. A vehicle may miss the data if the broadcast cycle is longer than $t_{i}$. Although reducing the broadcast cycle time can solve this problem, it reduces the DC and increases the network traffic. The second constraint is the bandwidth limit. Given a data broadcast cycle time, only a limited amount of data can be broadcast within one cycle. Disseminating data over this limit will cause collisions and data loss. As a result, the DC within the given D-Zone is equal to the maximum number of data items that can be broadcast to the D-Zone in one cycle, which is given by

$$
\mathrm{DC}=\frac{S \times T}{D_{\mathrm{avg}}}
$$

where $S$ denotes the throughput achievable by multihop broadcast in the D-Zone, $T$ denotes the broadcast cycle time, and $D_{\text {avg }}$ denotes the average data size.

Equation (1) clearly shows the two key factors that limit the DC of the DP scheme. First, the DP scheme reduces the broadcast throughput since many control messages are used to improve the data delivery ratio. Second, in a given D-Zone, the location that requires the shortest broadcast cycle time determines the overall broadcast cycle for the entire dissemination zone. However, vehicles moving on the other part of the road may not need such frequent broadcast, and vehicles moving along the A-Road indeed need a much lower broadcast frequency. Thus, a large amount of bandwidth is wasted in the DP scheme. These drawbacks motivate our design of the following scheme.

\section{C. $D P-I B$}

The DP-IB scheme follows the basic idea of the DP scheme, where the data are poured on the A-Road and vehicles on the C-Roads obtain the data when crossing the intersections. 
Instead of keeping the data on the A-Road, DP-IB only keeps the data at the intersections of the A-Road.

DP-IB relies on a simple device that is called relay and broadcast station (IBer) to improve the DC. An IBer can be the popular roadside units [21], [22] that are widely used in many VANETs or a simple computing device with a small amount of memory and a wireless card (e.g., IEEE 802.11b). These stand-alone IBers can be easily installed at the intersection. Since they are not required to connect to the wired network, the deployment cost is low. The IBer is used to buffer data copies and rebroadcast them periodically. As a result, the data center does not need to ensure delivering data to the end user. Instead, it only transmits data to those IBers on the A-Road. In other words, the data center does not have to frequently broadcast data to guarantee that the vehicles from C-Roads receive the data. The IBers ensure that vehicles passing the intersection can still obtain the data, although the frequency required to pour data from the data center is significantly reduced. Furthermore, the IBers can adapt different broadcast cycle times at different intersections, i.e., a longer cycle for a slow-moving traffic and a shorter cycle for a fast-moving traffic. Two issues still need further investigation.

1) How do we reliably upload the data from the data center to the IBers and then deliver it to the end users?

2) How do we choose different broadcast cycle times for different parts of the road?

Next, we address the first problem and leave the second problem to Section III.

1) Intersection Data Buffering and Rebroadcasting: The data center in DP-IB pours data on the A-Road using the R-DP scheme. When data are forwarded through the intersections, the IBers overhear the data and update their own buffers accordingly, i.e., insert new data item, update existing data to a new version, or remove the invalid data. The IBer will broadcast the updated data in the next broadcast cycle. Since the IBer uses a single-hop broadcast to deliver data to vehicles, the data broadcast throughput is high.

One problem may arise when a new data item from the data center is transmitted to the intersection while the IBer is in the middle of broadcasting its buffered data. It is possible that the transmission of the new data item collides with the IBer broadcast and that the IBer cannot receive the new data. Our solution is to have the IBer and the forwarding nodes alternatively obtain the channel to broadcast. More specifically, the IBer broadcast cycle is divided into two periods: In the first period (called busy period), the IBer broadcasts its buffered data, whereas the forwarding nodes temporarily hold their data. In the second period (the idle period), the forwarding nodes forward pending packets, whereas the IBer stops broadcasting and only listens to the channel for passing-by data packets. In this way, the IBer releases the channel for a period of time in every broadcast cycle so that new data items can be received.

Intersection Contention Avoidance Protocol: All vehicles switch between two modes: active and inactive forwarding modes. They stay in the active forwarding mode most of the time and only switch to inactive when they are inside an IBer broadcast range and the IBer is in the busy period at the same time. When the vehicle is in the active forwarding mode, it forwards data using the R-DP scheme. It switches to inactive immediately after receiving a broadcast data packet from an IBer. In the inactive forwarding mode, the vehicle stops forwarding data until it goes back to active.

The IBer broadcasts an IBer_Idle message when its busy period ends. All vehicles receiving this message switch to active forwarding and start to forward any pending data. A vehicle in the inactive forwarding mode may fail to receive the IBer_Idle message due to message loss, or it may move out of the IBer broadcast range when the busy period ends. Thus, the inactive forwarding mode is set as a soft state, and the vehicle switches back to active if it does not receive a broadcast packet from the IBer for a time period.

Determining the Busy/Idle Period: If an IBer does not buffer too much data, it can simply send an IBer_Idle message after broadcasting all its buffered data. However, if the amount of data buffered exceeds the data DC, the IBer should stop broadcasting before the end of the cycle and leave some time for the idle period. Equation (2) is used to determine the length of the busy period, i.e.,

$$
L_{\mathrm{est}}=\frac{n \times D_{\mathrm{avg}}}{B_{\mathrm{IBer}}}+\frac{\sum_{i=1}^{m} \frac{T}{I_{i}} \times D_{\mathrm{avg}}}{B_{\mathrm{R}-\mathrm{DP}}} .
$$

In (2), $n$ is the number of buffered data items, $D_{\text {avg }}$ is the average data size, $m$ is the number of data centers that have stored data at the IBer, and the IBer receives the data packet from the $i$ th data center every $I_{i}$ time interval. $B_{\mathrm{IBer}}$ and $B_{\mathrm{R}-\mathrm{DP}}$ represent the measured broadcast throughput of the IBer and the R-DP scheme, respectively. $T$ is the actual broadcast cycle of the IBer. The IBer can locally obtain all the aforementioned information. $L_{\text {est }}$ estimates the time needed to finish broadcasting all the buffered data and the time to overhear all the new data packet generated in a broadcast cycle. If $L_{\text {est }}$ is smaller than $T$, the IBer can finish broadcasting all the buffered data and then send the IBer_Idle message. The rest of the broadcast cycle still allows the IBer to overhear all the new data. When $L_{\text {est }}$ is larger than $T$, the IBer is only given $\alpha T$ in a cycle to broadcast data before sending out the IBer_Idle message, where $\alpha$ is a system parameter, and it is much smaller than 1 . In the rest of the broadcast cycle $(1-\alpha) T$, the IBer enters the idle period.

2) Data Update and Invalidation: When new data items are added or old data items are updated at the data center, the data center immediately broadcasts the new data or the invalidation message using the R-DP scheme. The data center may occasionally rebroadcast the data that have been disseminated, in case the IBer fails to obtain the data. Since all copies of the disseminated data are only buffered at the intersections along the A-Road, one invalidation message sent along the A-Road will be able to remove the invalid copies.

3) Data DC: The DC of DP-IB is limited by the broadcast cycles of the IBers. When compared with the DP scheme, the number of control messages in DP-IB is significantly reduced and, hence, can speed up the data transmission. Although an idle period is added to the broadcast cycle, it only takes a small portion of the broadcast cycle time, whereas the majority of the 
broadcast cycle time is spent on data broadcast. Thus, it has a better bandwidth utilization than DP, where each data packet transmission may involve many control messages and extra backoff times. As a result, the throughput of data broadcast in DP-IB is much higher than DP, and the DC is higher.

\section{AnALYZING AND DEtermining ThE BROADCAST CYCLE TIME}

In this section, we use an analytical model to determine the broadcast cycle time at the intersection, denoted as $T_{i} . T_{i}$ is used to determine the DC and the delivery ratio of the DP scheme and the DP-IB scheme. $T_{i}$ is also closely related to the time for a vehicle going through the intersection region, denoted as $t_{i}$. Intuitively, $T_{i}$ should be less than the minimum $t_{i}$ of all vehicles moving through the intersection region to guarantee that all vehicles passing the intersection can receive the broadcast data. If disseminating more data becomes the main objective, we can improve the DC by increasing $T_{i}$. This is at the cost of reducing the data delivery ratio, since some vehicles moving across the intersection may miss a part of the data. To derive $T_{i}$, we first model the intersection delay.

\section{A. Modeling the Intersection Delay}

Although there are many different intersection structures in reality, such as signalized, isolated, roundabout, etc., our intersection delay model only studies the vehicle delay at the signalized intersection with two crossing paths, because it can simplify the presentation and still show the relation between the network properties and the vehicle traffic properties. Our analysis can be easily extended to more complicated intersections, where the vehicle delay distribution is usually modeled by applying advanced transportation traffic theory or empirical traffic flow statistics, which is beyond the scope of this paper.

The delay $t_{i}$ can be represented by two parts [23]: the first part, which is referred to as the moving delay (denoted as $t_{m}$ ), is the ideal travel time if this vehicle does not meet any traffic control signal. It is equal to the moving distance at the intersection divided by the normal moving speed of the vehicle. The second part, which is referred to as the queuing delay (denoted as $t_{q}$ ), is the extra delay caused by the traffic signal, including deceleration delay, stopped delay, and acceleration delay. Their relations are given by

$$
t_{i}=t_{m}+t_{q}
$$

1) Calculating $t_{m}, t_{q}$, and $t_{i}: t_{m}$ is related to the vehicle moving distance and speed on the C-Road covered by the data broadcast. The broadcast node is always on the A-Road, and the broadcast can reach a fixed portion of the C-Road. Given the length of the C-Road covered by the broadcast as $R, t_{m}$ can be easily computed as

$$
t_{m}=R / v
$$

where $v$ is the normal speed of the vehicle moving across the intersection.

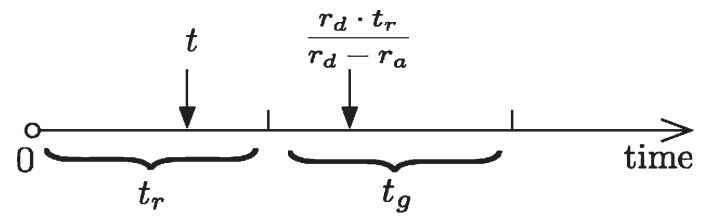

Fig. 2. Analytical model for $t_{q}$.

To obtain the minimum $t_{i}$, vehicles move through the intersection at its normal speed without deceleration and stop. Thus, $t_{i}^{\min }=t_{m}$. In reality, vehicles rarely keep the normal speed at the intersection because of traffic control signals. Most vehicles experience deceleration and acceleration and often wait in line with full stop [24]. Not all the vehicles experience the same delay when traveling through an intersection. The delay depends on a number of factors such as the traffic flow density, the signal time, and the time when the vehicle arrives at the intersection. Based on an example about 11 vehicles shown in [24], the first eight vehicles reaching the intersection come to a complete stop. These vehicles need to stop either as a consequence of their arrival during the red interval or during the green interval when the queue of vehicles that had formed during the previous red interval has not yet fully dissipated. It is further observed that the following three vehicles only experience deceleration and acceleration delays, as these vehicles reach the intersection when all previously queued vehicles have already started to move and therefore only need to slow down to maintain a safe distance from the vehicles ahead of them.

We study the intersection delay with undersaturated traffic flow, which means that the arrival rate $r_{a}$ is less than the saturated departure rate $r_{d}$, and we assume that the queue of vehicles formed during the red signal cycle can always be cleared before the next red signal.

Let $t_{r}$ and $t_{g}$ denote the red and green signal durations, respectively. Suppose a vehicle arrives at the intersection $t$ seconds after the red signal turns on. As shown in Fig. 2, the $t_{q}$ of this vehicle is given by

$$
t_{q}= \begin{cases}t_{r}-t+\frac{r_{a} \cdot t}{r_{d}}, & t \in\left[0, \frac{r_{d} \cdot t_{r}}{r_{d}-r_{a}}\right] . \\ 0, & \text { else }\end{cases}
$$

In (5), $r_{d} \cdot t_{r} /\left(r_{d}-r_{a}\right)$ shows the time it takes to clear the queue accumulated at the intersection after the red signal starts. When the vehicle arrives at the intersection after that time, it will go through the intersection without delay; if the vehicle arrives before that time, it waits for the vehicles that queued at the intersection to depart, and the delay can be calculated by (5).

We assume that the vehicle arrival follows a uniform distribution, which is widely used in traffic flow modeling [24]. The probability distribution function (pdf) of $t_{q}$ is given by

$$
\begin{aligned}
f_{t_{q}}(x)= & \operatorname{Prob}\left\{t_{q}=x\right\} \\
= & \begin{cases}0, & x<0 \\
1-\frac{r_{d} \cdot t_{r}}{\left(r_{d}-r_{a}\right)\left(t_{r}+t_{g}\right)}, & x=0 \\
\frac{r_{d}}{\left(r_{d}-r_{a}\right)\left(t_{r}+t_{g}\right)}, & 0<x \leq t_{r} \\
0, & x>t_{r}\end{cases}
\end{aligned}
$$


Since $t_{m}$ can be computed as a constant from (4), the pdf of $t_{i}$ (i.e., $f_{t_{i}}(t)$ ) can be easily computed by combining (3), (4), and (6), i.e.,

$$
f_{t_{i}}(x)=\operatorname{Prob}\left\{t_{i}=x\right\}=\operatorname{Prob}\left\{t_{q}=x-t_{m}\right\}=f_{t_{q}}\left(x-t_{m}\right) .
$$

\section{B. Determining the Broadcast Cycle Time}

It is possible to deliver all data in D-Set to every moving vehicles on the C-Road only when the data broadcast cycle time $T_{i}$ at the intersection is smaller than the minimum vehicle delay $t_{i}$. We define this broadcast cycle time, which allows all vehicles to receive all data in D-Set as full delivery broadcast cycle time, which is denoted by $\bar{T}_{i}$, and it is given by

$$
\bar{T}_{i} \leq t_{m}=R / v
$$

Suppose the transmission range of the broadcast is $100 \mathrm{~m}$, and the C-Road perpendicularly intersects with the A-Road. Then, the length of the C-Road covered by the broadcast $R=200 \mathrm{~m}$. Assume that the normal vehicle speed at the intersection is $45 \mathrm{mi} / \mathrm{h}(20.1 \mathrm{~m} / \mathrm{s})$. Then, $\bar{T}_{i}$ is less than $9.95 \mathrm{~s}$, that is, the broadcast node at the intersection has to finish broadcasting all data in the D-Set in $9.95 \mathrm{~s}$.

In simple DP and R-DP, all intersections on the A-Road and the data center use the same broadcast cycle time, which is computed by

$$
T_{\mathrm{DC}}=\min _{i \in \mathrm{A}-\mathrm{Road}}\left\{\bar{T}_{i}\right\} .
$$

Thus, the intersection that requires the minimum broadcast cycle time among all the intersections determines the broadcast cycle time. However, in DP-IB, different intersections use different broadcast cycle times. It is controlled by the local broadcast node IBer and can be adaptively adjusted based on the local vehicle traffic condition.

\section{Relations Between Delivery Ratio, Broadcast Cycle Time, and $D C$}

Equation (1) shows that the DC is linear to the broadcast cycle time. From the previous section, $\bar{T}_{i}$ sets the upper bound for the broadcast cycle time to ensure the best delivery ratio, which also bounds the maximum DC. By sacrificing some data delivery ratio, we can increase $T_{i}$ and broadcast more data. Next, we study the effects of $T_{i}$ on the data delivery ratio and explore the tradeoff between DC and data delivery ratio.

Given that the pdf of $t_{i}$, i.e., $f_{t_{i}}(x)$, can be computed by (7), the data delivery ratio at the intersection (denoted as DR) can be computed as

$$
\mathrm{DR}=\int_{T_{i}}^{\infty} f_{t_{i}}(x) d y+\int_{0}^{T_{i}} f_{t_{i}}(x) \cdot \frac{x}{T_{i}} d x .
$$

Equation (10) shows that vehicles staying longer than $T_{i}$ at the intersection (i.e., $t_{i}>T_{i}$ ) can receive all the broadcasted data,

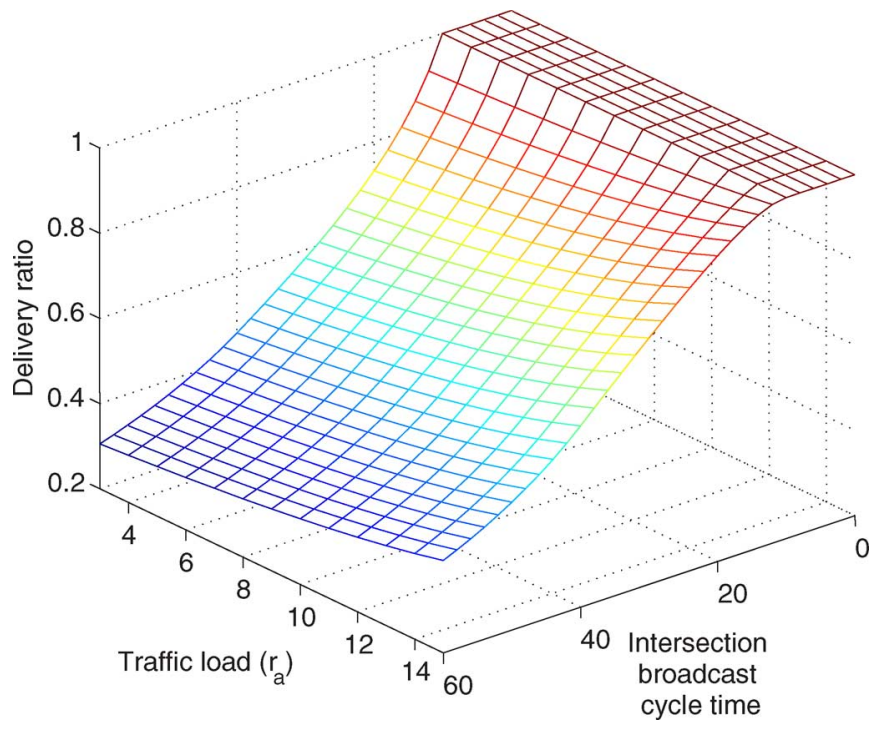

Fig. 3. Analysis of the relation between the broadcast cycle time and the data delivery ratio.

whereas those staying shorter than $T_{i}$ (i.e., $t_{i}<T_{i}$ ) only receive $t_{i} / T_{i}$ portion of the broadcasted data.

By combining (6), (7), and (10), the relation between DR and $T_{i}$ can be derived as

$\mathrm{DR}=\left\{\begin{array}{ll}\frac{1,}{t_{m}\left(\left(r_{d}-r_{a}\right)\left(t_{r}+t_{g}\right)-r_{d} t_{r}-\frac{1}{2} r_{d} t_{m}\right)} & T_{i} \leq t_{m} \\ \left(r_{d}-r_{a}\right)\left(t_{r}+g_{g}\right) T_{i} & \\ +\frac{r_{d}\left(t_{m}+t_{r}\right)-\frac{1}{2} r_{d} T_{i}}{\left(r_{d}-r_{a}\right)\left(t_{r}+t_{g}\right)}, & t_{m}<T_{i} \leq t_{m}+t_{r} . \\ \frac{2 t_{m}\left(t_{r}+t_{g}\right)\left(r_{d}-r_{a}\right)+r_{d} t_{r}^{2}}{2 T_{i}\left(r_{d}-r_{a}\right)\left(t_{r}+t_{g}\right)}, & T_{i}>t_{m}+t_{r}\end{array}\right.$.

In (11), the signal lengths $t_{g}$ and $t_{r}$ can be seen as fixed road properties. Parameter $r_{d}$ represents the capability of the intersection to disperse vehicle traffic, which is usually fixed after the road has been constructed. Parameter $r_{a}$ indicates the dynamic vehicle traffic load at the intersection. Therefore, with $t_{g}=30 \mathrm{~s}, t_{r}=30 \mathrm{~s}, t_{m}=10 \mathrm{~s}$, and $r_{d}=30$, the relations among $T_{i}$, DR, and the local vehicle traffic load are shown in Fig. 3.

Fig. 3 clearly shows that the DC and the data delivery ratio are conflict design goals. If we are given the delivery ratio requirement, for example, DR $>90 \%$, we can compute the largest $T_{i}$ based on (11). This $T_{i}$ value provides the maximum DC while keeping the delivery ratio equal to $90 \%$. Using the aforementioned relations, we can tradeoff these two metrics for the best broadcast strategy based on the requirement of the applications. This figure also addresses the second question raised in Section II-C1. The figure shows the relations among the broadcast cycle time, the data delivery ratio, and the vehicle traffic load. Generally speaking, when $T_{i}$ increases, the data delivery ratio drops. As the vehicle traffic density increases, vehicles stay longer at the intersection because they move slower and stop longer. Thus, more data can be delivered to drive-through vehicles by extending the broadcast cycle time at the intersection without reducing the data delivery ratio. Also, if the passing-through vehicles only receive a part of the D-Set without changing the broadcast cycle time, the dissemination 


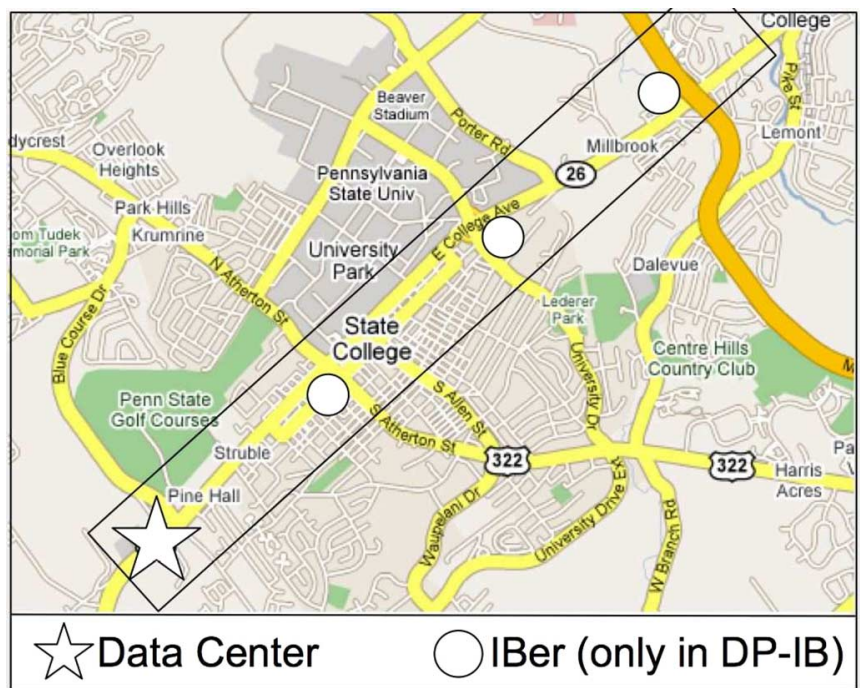

Fig. 4. Snapshot of the simulation setup area.

ratio will be higher as the vehicle traffic density increases. This provides guidelines for DP-IB to dynamically adapt its broadcast cycle time based on the local vehicle traffic load at different intersections.

\section{Performance Evaluations}

In this section, we evaluate the performance of the simple DP scheme (without using techniques presented in Section II-B2), the R-DP scheme, and the DP-IB scheme. We also compare these schemes to the OD scheme presented in [11] and the MAC-layer-based reliable multihop broadcast scheme (UMB) proposed in [16].

\section{A. Simulation Model}

We developed an ns-2 [25]-based simulator to evaluate the proposed schemes. The simulation is based on a $4500-\mathrm{m} \times$ 600-m rectangle area extracted from a real street map of State College, PA. The positions of the data center and the D-Zone, which are represented by the star and the crossing rectangle, respectively, are shown in Fig. 4. We choose East College Avenue, which is one of the most crowded streets in State College, as the A-Road. It runs through the downtown with a $25 \mathrm{mi} / \mathrm{h}$ speed limit. The C-Roads are selected from three major streets that intersect with the East College Avenue. All the three C-Roads have the same speed limit $(45 \mathrm{mi} / \mathrm{h})$. The street layout and speed limit information are translated into a text format map, which meets the specification of Topologically Integrated Geographic Encoding and Referencing database [26] from the U.S. Census Bureau. These text-based map data are then transformed into the data format that can be used by ns-2, based on techniques presented in [27]. To simulate the vehicle traffic on the A-Road, we initially randomly deploy 150 vehicles on the A-Road and let them move toward either end of the road. Those vehicles move back and forth with $25 \mathrm{mi} / \mathrm{h}$ during the simulation to mimic a continuous traffic flow along the A-Road. We assume that the vehicle density on the A-Road is large enough to

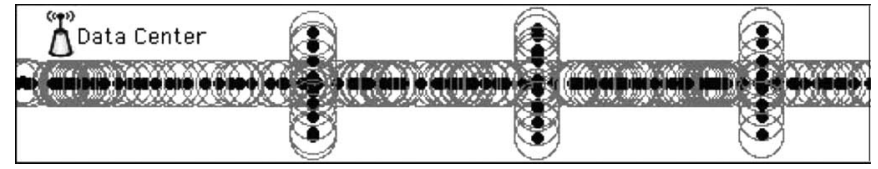

Fig. 5. Snapshot of the simulation setup area.

maintain network connection, which is possible in urban areas for which our data dissemination scheme is designed.

Since vehicles that move along the A-Road can always receive the disseminated data, we are more interested in vehicles on C-Roads. Among vehicles on the C-Roads, only those close to the intersections are relevant to data dissemination. Since simulating the movement of vehicles across the intersection is more important than the traffic beyond the intersection, we only consider the vehicle traffic on a 600-m-long section of C-Roads, whose middle point intersects with the A-Road. We initially deploy 20 nodes at the upper end of each C-Road and let them move back and forth between the two ends of the C-Roads. When the node arrives at the intersection, it stops for a random amount of time with the distribution given by (6) before moving again. When the node arrives at the other end of the road section, it pauses for a time period and moves back. Each vehicle on C-Roads randomly picks a value between 15 and $45(\mathrm{mi} / \mathrm{h})$ as its moving speed. Fig. 5 shows a snapshot of the simulation area. The data center is located at the left end of the A-Road and disseminates data along the A-Road toward the right.

Simple DP and R-DP use the same broadcast cycle time throughout the A-Road. DP-IB can adapt the IBer broadcast cycle time based on the local vehicle traffic at the intersection. In our simulation model, the same traffic pattern is used in all the C-Roads, and hence, we use the same intersection broadcast cycle time for all intersections.

At the data center, all data in the D-Set are repeatedly injected to the A-Road. The data items are sent by the data center one after another with a given time interval. In simple DP and R-DP, this time interval is equal to the broadcast cycle time divided by the number of data items to be disseminated. In DP-IB, since the frequency required to pour data from the data center can be greatly reduced, it takes much longer to broadcast the whole data set at the data center. Thus, the time interval is set as $250 \mathrm{~ms}$. We only consider data items with a fixed size of 2500 bits. Each vehicle sends a beacon message every $0.5 \mathrm{~s}$ to report its own location and speed.

Most experiment parameters are listed in Table I. The performance of the protocols is measured by the following two metrics.

- Data delivery ratio: For each vehicle, the data delivery ratio is the total number of the received nonidentical data items divided by the total number of the disseminated data items.

- Network traffic overhead: The number of bits generated per second, which is a summation of individual packethops. For instance, if a packet of 1000 bits is forwarded ten hops, the network traffic overhead is counted as $10 \mathrm{~K}$ bit-hops. 
TABLE I

SIMULATION SETUP

\begin{tabular}{|l|c|}
\hline Parameter & Value \\
\hline Simulation time & 500 seconds \\
\hline Simulation area & $4500 \mathrm{~m} \times 600 \mathrm{~m}$ \\
\hline Communication range & $100 \mathrm{~m}$ \\
\hline Number of nodes on the A-Road & 150 \\
\hline Number of nodes on the C-Roads & 60 \\
\hline Vehicle velocity & $15-45 \mathrm{miles}$ per hour \\
\hline Data packet size & 2500 bit \\
\hline Dissemination data set size & $10-600$ data items \\
\hline Bandwidth & $10 \mathrm{Mbps}$ \\
\hline Intersection broadcast cycle time & $5-40 \mathrm{~s}$ \\
\hline Beacon interval & 2 beacon/second \\
\hline Beacon packet size & $512 \mathrm{bit}$ \\
\hline Control packet size & $512 \mathrm{bit}$ \\
\hline DP-IB cache replacement policy & FIFO \\
\hline
\end{tabular}

For each measurement, 30 simulation runs are used, and a different seed value is used for each simulation run. For the data delivery ratio, the mean value of the measured data is obtained by collecting a large number of samples such that the confidence interval is reasonably small. In most cases, the $95 \%$ confidence interval for the measured data is less than $10 \%$ of the sample mean.

\section{B. Simulation Results}

1) Relation Between Delivery Ratio and DC: Fig. 6 shows the relation between delivery ratio and the amount of data to be disseminated (DC) for the five schemes. When only a small amount of data items are disseminated (e.g., ten data items in Fig. 6), there is plenty of available bandwidth, and the data delivery ratios of all schemes are close to $100 \%$. Among them, the OD scheme has a slightly higher delivery ratio than the others because OD explores every possible path to propagate the data. However, the delivery ratio of OD becomes much lower than that of DP and DP-IB when more data items (above 50) are disseminated. As discussed in Section I, the OD scheme generates too much redundant network traffic, which may lead to severe congestion and significantly reduce the data delivery ratio. When the D-Set size is above 50, there is a significant increase of data losses in the simple DP scheme, and its data delivery ratio drops dramatically. Therefore, the DC of the simple DP scheme will be very low to maintain a good data delivery ratio.

UMB, R-DP, and DP-IB have very high data delivery ratios when the data set size is below 150 . When more data are to be disseminated, the delivery ratios of the UMB scheme and the R-DP scheme drop quickly, whereas the DP-IB scheme keeps the same data delivery ratio. The data delivery ratio of UMB drops sharply because the packets in a dissemination data flow interfere with each other. As the channel usage increases, the interference problem becomes worse, leading to severe packet loss. Although UMB can effectively improve the reliability of multihop broadcast, this MAC-layer approach cannot address network congestion. The low delivery ratio of R-DP is not because of packet loss since the packet can still be reliably transmitted. Thus, the delivery ratio of R-DP drops gracefully when compared to that of UMB. However, the R-DP scheme

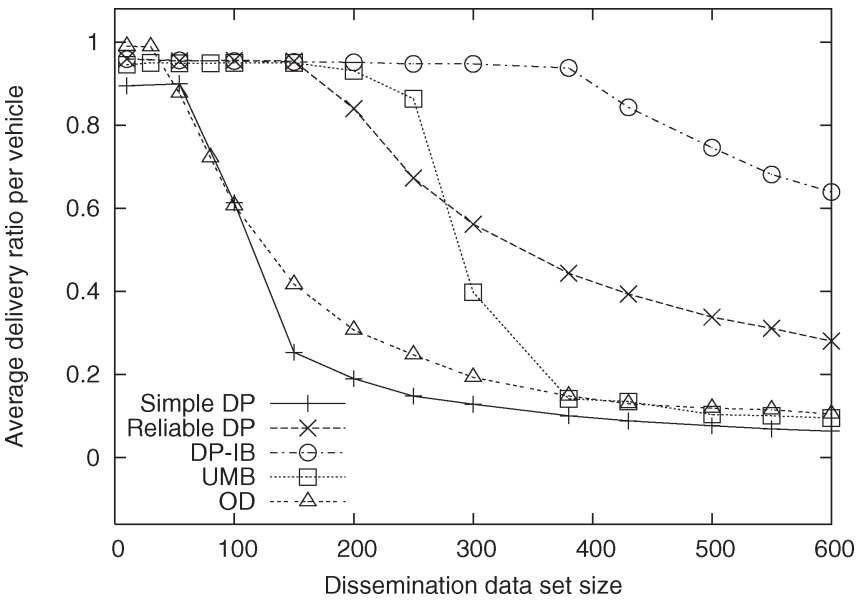

Fig. 6. Comparison of the data delivery ratio (with a broadcast cycle of $10 \mathrm{~s}$ ).

reaches the saturate status when the D-Set size reaches 150 . When more data need to be disseminated, R-DP cannot finish broadcasting the whole data set within one cycle. As a result, some vehicles move across the intersection without receiving all the data. DP-IB can broadcast data with a higher throughput and will not reach the saturate state until 360 data items are broadcast. Thus, it has more DC than the R-DP scheme. When the D-Set size is more than 360 , the IBer in the DP-IB scheme may not be able to finish broadcasting the whole data set within its broadcast cycle time, and the data delivery ratio decreases.

2) Revisiting the Relations Between Delivery Ratio, Broadcast Cycle Time, and DC: Fig. 6 only shows the results under a fixed broadcast cycle time (10 s). To further examine the relations among the broadcast cycle $T_{i}$, the data delivery ratio, and the DC, we present more results in Fig. 7 when the D-Set size and the broadcast cycle time change. Fig. 7 shows additional relations that are not shown in Fig. 3, which does not consider the network bandwidth limit. In Fig. 3, we assume that the whole D-Set can be transmitted. If vehicles fail to receive the data, it is because the broadcast cycle time is too long and the vehicles passes the intersection before one cycle finishes (short intersection stay). However, data may not be delivered because the D-Set set is too large. That is, the network is saturated and cannot accommodate all the disseminated data.

Fig. 7 studies the packet loss caused by both factors, namely short intersection stay and network saturation, under the same vehicle traffic load. In Fig. 7, the 3-D surface in each figure can be divided into four regions, based on the factors causing the packet loss. As shown in Fig. 7(a), in Region 1, there is no packet loss because of the aforementioned factors, and the delivery ratio is close to $100 \%$. In this region, the broadcast cycle time is smaller than $10 \mathrm{~s}$. Then, there is no packet loss due to short intersection stay since even the fastest vehicle takes $10 \mathrm{~s}$ to move across the intersection. Also, the D-Set size is small, and the network is not saturated. In Region 2, the only factor for packet loss is network saturation since the D-Set cannot be delivered within $10 \mathrm{~s}$. There is no packet loss due to short intersection stay as the broadcast cycle time is still less than $10 \mathrm{~s}$. In Region 3, the packet loss is due to short intersection stay since the broadcast cycle time is longer than $10 \mathrm{~s}$, and some vehicles may pass the intersection without getting the data. 


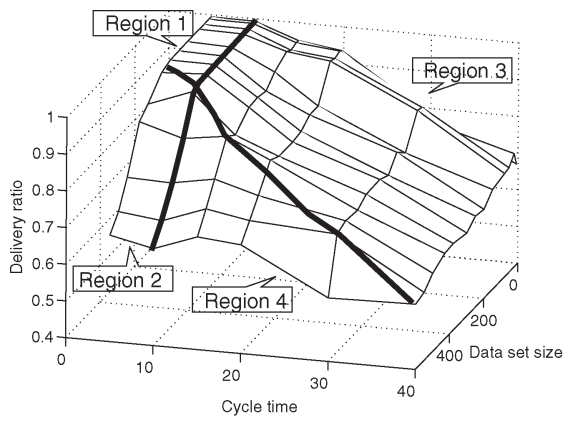

(a)

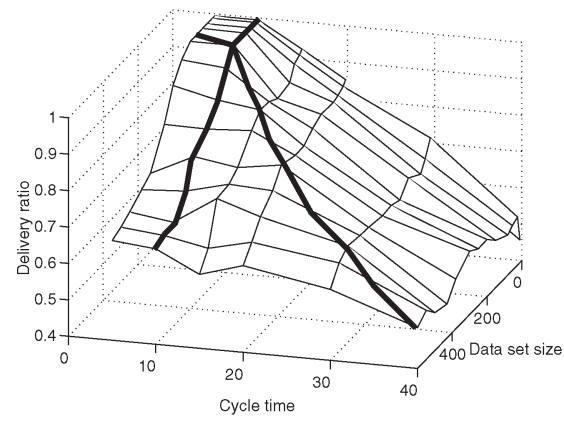

(b)

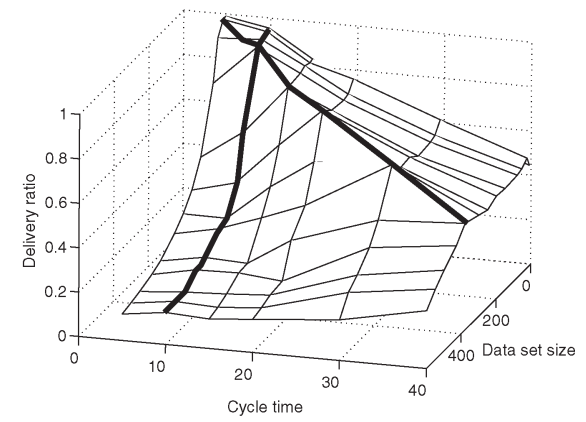

(c)

Fig. 7. Relations among cycle time, data set size, and delivery ratio. (a) DP-IB. (b) R-DP. (c) Simple DP.

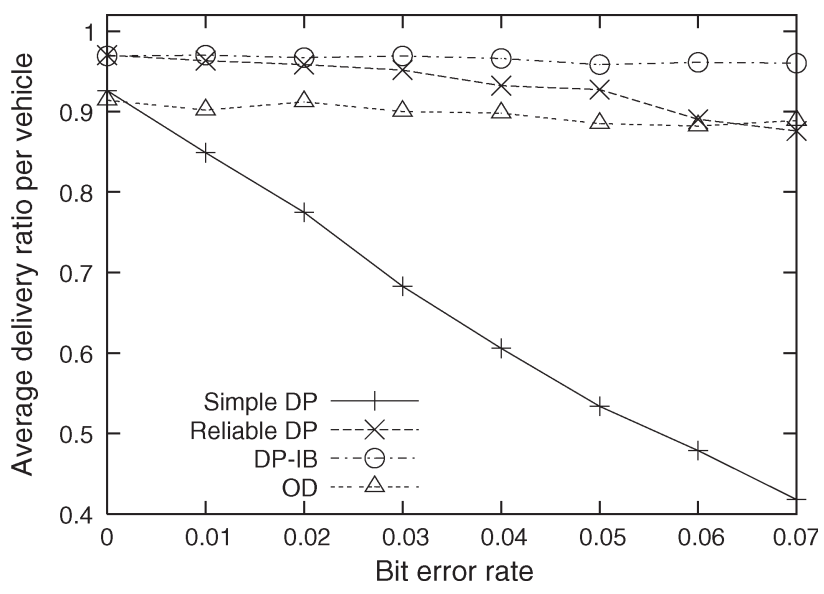

Fig. 8. Impact of the transmission bit error rate.

There is no packet loss due to network saturation because the D-Set can be sent within the given broadcast cycle time. Thus, within this region, the D-Set size can be increased without affecting the data delivery ratio. In Region 4, the D-Set size exceeds the network capacity, and the broadcast cycle time is longer than $10 \mathrm{~s}$; thus, packets are lost for both factors.

Fig. 7(b) and (c) can also be divided into four regions using the same criteria, but the areas of the same region are different in these three schemes. The areas of Region 1 and Region 3 in DP-IB are much larger and flatter than the other schemes. It means that DP-IB can always disseminate the largest amount of data without reaching network saturation. In simple DP, there is no mechanism to reduce the collision, so the bandwidth utilization is very low. Therefore, the areas of its Region 2 and Region 4 are very large, whereas the areas of its Region 1 and Region 3 are quite small. In most cases, the network is saturated, and less data can be successfully transmitted without collision or being dropped.

Overall, given any required data delivery ratio, DP-IB can always disseminate the largest amount of data when a proper broadcast cycle time is chosen. R-DP can disseminate less data than DP-IB but still far more than the simple DP scheme under any data delivery ratio requirement.

3) Effects of Bit Error Rate: Fig. 8 compares the four schemes with different transmission bit error rates when 50 data items are disseminated. The simple DP scheme is based on simple broadcast, so the data delivery ratio quickly drops when the error rate increases. The data delivery ratio of the R-DP

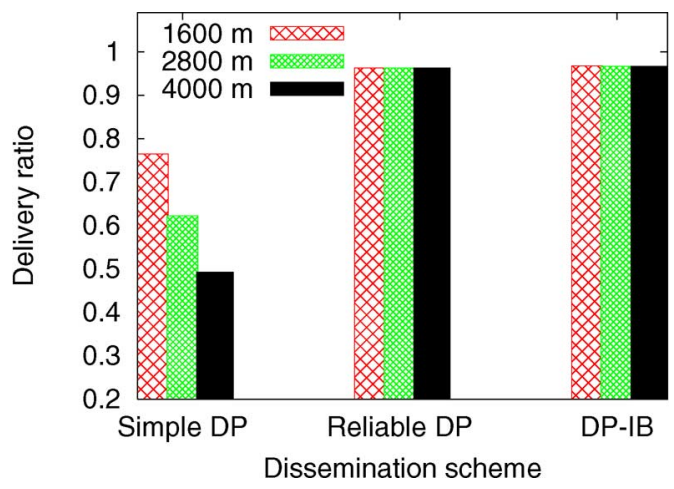

Fig. 9. Delivery ratio at different distances from the data center.

scheme only drops slightly when the error rate increases, which verifies the effectiveness of the reliable multihop broadcast. The OD scheme can also keep a good delivery ratio due to its opportunistic nature. Among the four schemes, DP-IB is the most resilient to bit errors. Since the data are buffered at the intersection, the impact of bit error on multihop transmission is minimized.

4) Dissemination Distance: Fig. 9 shows the data delivery ratio of the three DP schemes when the data are delivered to vehicles far away from the data center. The D-Set size is 100 . As can be seen, the delivery ratios of both R-DP and DP-IB are not affected by the dissemination distance. This also verifies the effectiveness of the reliable multihop broadcast scheme in handling transmission errors. On the other hand, the delivery ratio of the simple DP scheme drops quickly as the distance increases, because packet loss occurs at every hop and not much data are left after a long-distance pouring.

5) Dissemination Delay: Fig. 10 shows the delay of delivering a new data item to vehicles at three intersections using the UMB scheme and the three DP schemes. The D-Set size is set to 50. The delay is computed from a new version of a data item generated at the data center to the time when the first vehicle receives it at the specific intersection. As shown in the figure, UMB has the lowest delay since it does not block the data flow and does not use any timers. The simple DP scheme does not use any reliable transmission mechanism, but the delay is still longer than UMB because some new data items fail to reach the intersection in the first cycle after being generated. When it is delivered in the second cycle, the delay is increased by one cycle time (10 s). The R-DP scheme has a higher delay 


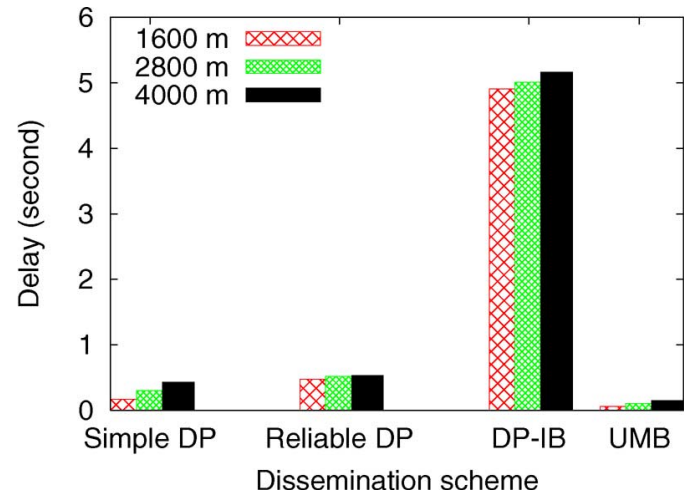

Fig. 10. Delivery delay at different distances from the data center.

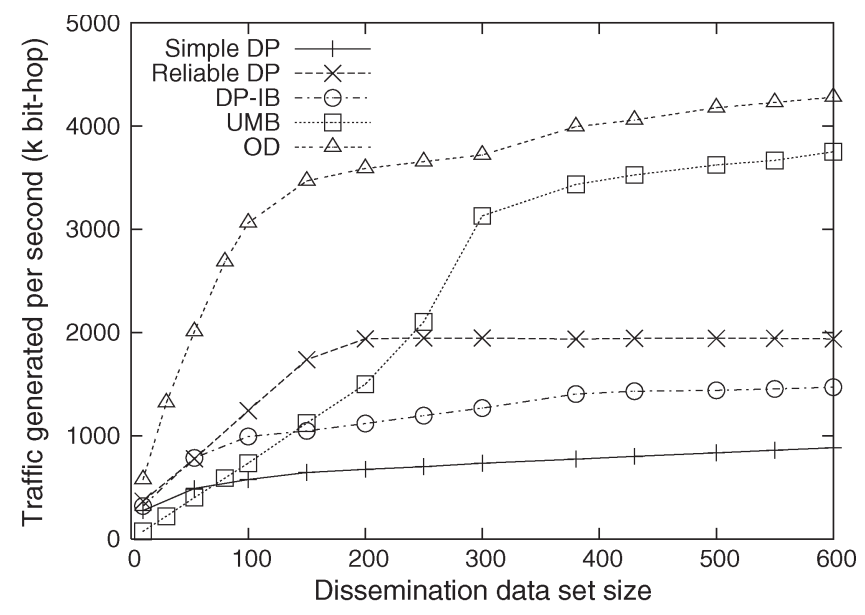

Fig. 11. Comparison of the network traffic.

since it blocks the flow until the ACK is received for the previous packet, and this increases the packet forwarding latency. DP-IB has the highest delay due to the following reason: after a new data item has been generated, it needs to be uploaded to the IBer, and the upload only starts after the IBer finishes its broadcast cycle. Hence, the new data item needs to wait for half of the broadcast cycle time on average before transmitting the data to the IBer. However, the delay is at the level of seconds and only happens when the accessed data item is updated. Most of the time, this delay does not exist. Thus, this dissemination delay should not be a big issue when compared to the other factors such as DC and data delivery ratio.

6) Network Traffic Overhead: Fig. 11 compares the overall network traffic overhead generated by the five schemes when the D-Set size increases. The simple DP scheme generates the least amount of traffic since it simply broadcasts the data without adding any control message. Furthermore, many data packets are dropped before going too far on the A-Road. This can be reflected by the low data delivery ratio, as shown in Fig. 6.

The OD scheme has the highest traffic overhead since vehicles keep sending data to each other. The traffic overhead of the R-DP scheme is also pretty high because many control messages are generated for each data packet transmission, and it introduces many retransmissions to improve the reliability.

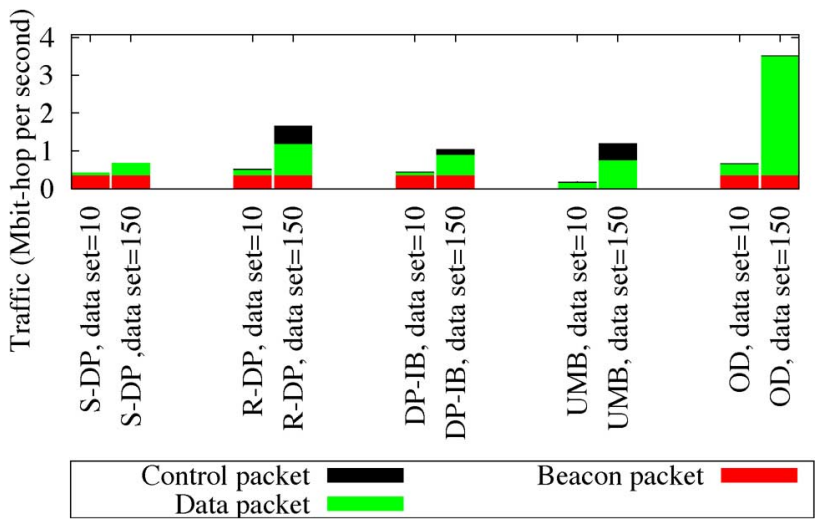

Fig. 12. Detailed comparison of the network traffic.

DP-IB generates much less traffic when compared to R-DP, since DP-IB delivers data to end nodes by a single-hop broadcast from the IBers instead of a reliable multihop broadcast from the data center. As a result, the data center broadcasts data at a much lower rate, which greatly reduces the network traffic overhead.

These five schemes generate more network traffic when the D-Set size increases at the beginning. After the channel saturates, the disseminated data may be dropped, and the traffic only slightly grows or even keeps constant when the number of the dissemination data increases. Fig. 11 shows that the network traffic of the R-DP scheme keeps almost constant and DP-IB only slightly grows when they disseminate more data than their DCs, which are 150 and 360 data items, respectively. Fig. 11 also shows that when the channel saturates, DP-IB disseminates more data than R-DP but still generates less traffic, which verifies that DP-IB can improve the bandwidth utilization.

Fig. 12 shows the distribution of the network traffic. The control packet includes the beacon packets used by all schemes except UMB, the RTS/CTS handshake used by R-DP and DP-IB, and the control packets for the intersection contention avoidance used by DP-IB. In the figure, all schemes generate the same amount of beacon messages except UMB. As can be seen, all schemes have a very small amount of control packets when the network traffic load is low (e.g., to disseminate ten data items). When the network traffic load increases (e.g., to disseminate 100 data items), the portion of control packets becomes significant for the R-DP scheme. On the other hand, the control traffic of the DP-IB scheme is not very high because the data center can slow down the rate of pushing data to the intersections. For the OD scheme, although it does not have control traffic overhead, its data packet overhead is much higher. As explained earlier, the simple DP scheme drops many packets, and hence, the traffic overhead is lower than the others.

\section{CONCLuSion AND Future Work}

VANETs have been envisioned to provide increased convenience and efficiency to drivers on the road. To realize this vision, we proposed a DP and buffering paradigm to address the data dissemination problem in a VANET. In DP, data are 
periodically broadcast to vehicles on the road. In DP-IB, data poured from the data center are buffered and rebroadcast at the intersections. Simulation results show that the proposed DP-IB scheme can significantly improve data delivery ratio and reduce network traffic. Furthermore, we provide analytical models to explore the DC of the proposed schemes. The analytical models also provide guidelines on choosing the system parameters to maximize the DC under different data delivery ratio requirements.

To the best of our knowledge, this is the first paper to study DC in a VANET. The DP-IB scheme is proposed as a general solution for maximizing the DC, where different broadcast cycle times are used at the data center and different intersections. As stated in our system model, the DP scheme is designed for environments where the vehicle density is reasonably high to maintain connection. Since the DP scheme is only used to deliver the data from the data center to the intersection, it can be replaced by other schemes without significantly affecting the DC. Thus, in a sparse environment, store and forward solutions [28] and caching techniques [29] can be added to the DP scheme to deal with disconnections.

In this paper, we focused on push-based data dissemination, where the data can be efficiently delivered from moving vehicles or fixed stations to other vehicles. In our previous work [28], we studied the pull-based data dissemination/access, where a vehicle is enabled to query information about specific targets. Generally speaking, the push-based approach is used to disseminate data that are useful for many people, whereas the pull-based approach is used to query data that are specific for some user. In practice, a hybrid of push/pull can be used to improve the system performance, and this will be studied in our future work.

\section{REFERENCES}

[1] M. D. Dikaiakos, S. Iqbal, T. Nadeem, and L. Iftode, "VITP: An information transfer protocol for vehicular computing," in Proc. ACM VANET, 2005, pp. 30-39.

[2] T. Mak, K. Laberteaux, and R. Sengupta, "A multi-channel VANET providing concurrent safety and commercial services," in Proc. ACM VANET, Sep. 2005, pp. 1-9.

[3] X. Yang, J. Liu, F. Zhao, and N. Vaidya, "A vehicle-to-vehicle communication protocol for cooperative collision warning," in Proc. Int. Conf. MobiQuitous, Aug. 2004, pp. 114-123.

[4] P. Deolasee, A. Katkar, A. Panchbudhe, and K. Ramamritham, "Adaptive push-pull: Disseminating dynamic web data," in Proc. WWW Conf., 2001, pp. $265-274$

[5] G. Muhl, A. Ulbrich, K. Herrmann, and T. Weis, "Disseminating information to mobile clients using publish-subscribe," IEEE Internet Comput., vol. 8, no. 3, pp. 46-53, May/Jun. 2004.

[6] V. Namboodiri, M. Agarwal, and L. Gao, "A study on the feasibility of mobile gateways for vehicular ad-hoc networks," in Proc. ACM VANET, Oct. 2004, pp. 66-75.

[7] V. Naumov, R. Baumann, and T. Gross, "An evaluation of inter-vehicle ad hoc networks based on realistic vehicular traces," in Proc. ACM MobiHoc, 2006, pp. 108-119.

[8] H. Wu, R. Fujimoto, R. Guensler, and M. Hunter, "MDDV: A mobilitycentric data dissemination algorithm for vehicular networks," in Proc. 1st ACM VANET, 2004, pp. 47-56.

[9] S. Ni, Y. Tseng, Y. Chen, and J. Sheu, "The broadcast storm problem in a mobile ad hoc network," in Proc. ACM Mobicom, 1999, pp. 151-162.

[10] D. Choffnes and F. Bustamante, "An integrated mobility and traffic model for vehicular wireless networks," in Proc. ACM VANET, 2005, pp. 69-78.

[11] B. Xu, A. Ouksel, and O. Wolfson, "Opportunistic resource exchange in inter-vehicle ad hoc networks," in Proc. IEEE Int. Conf. MDM, 2004, pp. 4-12.
[12] A. Demers, D. Greene, C. Hauser, W. Irish, J. Larson, S. Shenker, H. Sturgis, D. Swinehart, and D. Terry, "Epidemic algorithms for replicated database maintenance," Oper. Syst. Rev., vol. 22, no. 1, pp. 8-32, 1988

[13] Z. Haas, J. Halpern, and L. Li, "Gossip-based ad hoc routing," in Proc. IEEE Infocom, 2002, pp. 1707-1716.

[14] G. Karlsson, V. Lenders, and M. May, "Delay-tolerant broadcasting," in Proc. SIGCOMM Workshop CHANTS, Pisa, Italy, 2006, pp. 197-204.

[15] Wireless LAN Media Access Control (MAC) and Physical Layer (PHY) Specifications, IEEE Std. 802.11, 1999. [Online]. Available: http://grouper.ieee.org/groups/802/11

[16] G. Korkmaz, E. Ekici, F. Ozguner, and U. Ozguner, "Urban multi-hop broadcast protocol for inter-vehicle communication systems," in Proc. ACM VANET, Oct. 2004, pp. 76-85.

[17] D. Goodman, J. Borras, N. Mandayam, and R. Yates, "INFOSTATIONS: A new system model for data and messaging services," in Proc. IEEE VTC, Rome, Italy, May 1997, vol. 2, pp. 969-973.

[18] Q. Xu, T. Mark, J. Ko, and R. Sengupta, "Vehicle-to-vehicle safety messaging in DSRC," in Proc. ACM VANET, Oct. 2004, pp. 19-28.

[19] J. Yin, T. Eibatt, G. Yeung, B. Ryu, S. Habermas, H. Krishnan, and T. Talty, "Performance evaluation of safety applications over DSRC vehicular ad hoc networks," in Proc. ACM VANET, Oct. 2004, pp. 1-9.

[20] G. Cao, "A scalable low-latency cache invalidation strategy for mobile environments," IEEE Trans. Knowl. Data Eng., vol. 15, no. 5, pp. 12511265, Sep./Oct. 2003

[21] A. Nandan, S. Das, G. Pau, M. Gerla, and M. Sanadidi, "Co-operative downloading in vehicular ad-hoc wireless networks," in Proc. 2nd Annu. Conf. WONS, 2005, pp. 32-41.

[22] Y. Zhang, J. Zhao, and G. Cao, "On scheduling vehicle-roadside data access," in Proc. ACM VANET, 2007, pp. 10-19.

[23] Transportation Research Board (2000), Highway Capacity Manual (2000), Washington, DC: Nat. Res. Council.

[24] F. Dion, H. Rakha, and Y. Kang, "Comparison of delay estimates at undersaturated and over-saturated pre-timed signalized intersections," Transp. Res., Part B: Methodol., vol. 38B, no. 2, pp. 99-122, Feb. 2004.

[25] The Network Simulator. [Online]. Available: http://www.isi.edu/ nsnam/ns/

[26] Tiger, Tiger/Line, and Tiger-Related Products, Washington, DC: U.S. Census Bureau. [Online]. Available: http://www.census.gov/geo/ www/tiger/

[27] A. K. Saha and D. B. Johnson, "Modeling mobility for vehicular ad hoc networks," in Proc. ACM VANET, Philadelphia, PA, Oct. 2004, pp. 91-92.

[28] J. Zhao and G. Cao, "VADD: Vehicle-assisted data delivery in vehicular ad hoc networks," in Proc. IEEE Infocom., 2006.

[29] L. Yin and G. Cao, "Supporting cooperative caching in ad hoc networks," IEEE Trans. Mobile Comput., vol. 5, no. 1, pp. 77-89, Jan. 2006.

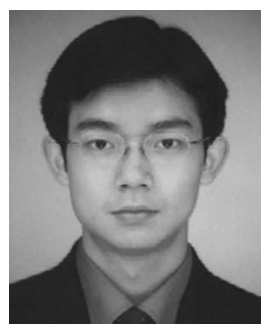

Jing Zhao (S'07) received the B.S. degree from Peking University, Beijing, China. He is currently working toward the Ph.D. degree in computer science and engineering at The Pennsylvania State University, State College.

His research interests include wireless networks, distributed systems, and mobile computing, with a focus on mobile ad hoc networks. 


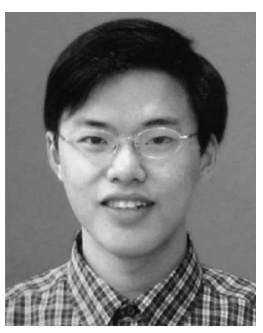

Yang Zhang (S'06) received the B.S. and M.E. degrees from Nanjing University, Nanjing, China, in 2002 and 2005, respectively. He is currently working toward the Ph.D. degree at The Pennsylvania State University, State College.

His research interests include distributed systems, mobile computing, and vehicular ad hoc networks.

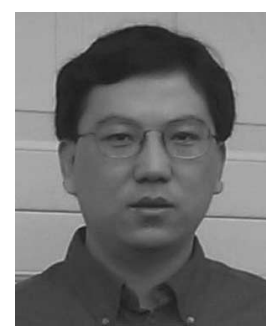

Guohong Cao (SM'07) received the B.S. degree from Xian Jiaotong University, Xian, China, and the M.S. and Ph.D. degrees in computer science from The Ohio State University, Columbus, in 1997 and 1999 , respectively.

Since 1999, he has been with the Department of Computer Science and Engineering, The Pennsylvania State University, State College, where he is currently an Associate Professor. His research interests are wireless networks and mobile computing. He has published more than 100 papers in the areas of sensor networks, wireless network security, data dissemination, resource management, and distributed fault-tolerant computing.

Prof. Cao is the Editor of the IEEE TRANSACTIONS ON Mobile COMPUTING and the IEEE TRANSACTIONS ON WIRELESS COMMUNICATIONS.

$\mathrm{He}$ has served on the program committee of many conferences. He was a recipient of the CAREER Award from the National Science Foundation in 2001. 